\title{
Determination of Physical Education and Sports Teachers' Nutrition Habits and Physical Activity Levels in the Global Epidemic (Covid-19) Process
}

\author{
Ramazan Erdogan ${ }^{1}$ \\ Metin Yilmaz ${ }^{2}$ (D) \\ Isa Aydemirs (D)
}

Bitlis Eren University School of Physical Education and Sport, Bitlis, Turkey. Email: ramaznerdogan@hotmail.com

${ }_{2,3}^{2,}$ Frat University Faculty of Sport Sciences, Elazig, Turkey.

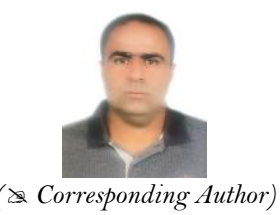

\section{Abstract}

This research was conducted to determine Physical Education and Sports teachers' nutritional habits and physical activity levels during the global epidemic (covid-19) process. 156 (121 male, 35 female) volunteer physical education and sports teachers working in Elazig province participated in the study. In order to determine the demographic information of participants, the personal information form; to determine the nutritional habits and physical activity levels, the "Investigation of Nutrition Habits and Nutrition Knowledge Levels" developed by Yücel (2015) and to determine the physical activity levels, the short form of the "International Physical Activity Questionnaire" were applied to participants. The SPSS package program was used in the analysis of the data and the significance level was accepted as $p<0.05$. According to the research results, it was determined that $89.1 \%$ of physical education and sports teachers did not receive nutrition education, $62.2 \%$ had a chronic disease, $27.6 \%$ were in the age range 43 and above, $67.3 \%$ were married, $59 \%$ were between $171-180 \mathrm{~cm}, 38.5 \%$ were between $71-80 \mathrm{~kg}, 27.6 \%$ had a seniority between $6-10$ years and $42,9 \%$ had income between $4001-5000$ TL. $47.4 \%$ of the teachers stated the effect of the state's "Stay at Home" project on physical activity as no, $37.2 \%$ yes, $15.4 \%$ partially and most of them stated that body weight increased during the pandemic period. It was observed that the mean scores of the participants' nutritional habits before the pandemic were lower than their mean scores during the pandemic period and a statistically significant difference was determined $(\mathrm{p}<0.05)$. In addition, before the pandemic, it was seen that the physical activity score averages of the participants were higher than the physical activity score averages during the pandemic period, and statistically significance was determined $(\mathrm{p}<0.05)$. As a result, it was observed that the eating habits of the physical education and sports teachers participating in the study were negatively affected during the pandemic period, and their physical activity levels decreased. In this context, we believe that the promotion of regular physical activities as well as adequate and balanced nutrition during the epidemic period will contribute to the individuals to overcome the epidemic with minimum damage.

Keywords: Physical education Sports teacher, Coronavirus, Healthy lifestyle, Nutrition habits, Physical activity.

Citation | Ramazan Erdogan; Metin Yilmaz; Isa Aydemir (2021). Determination of Physical Education and Sports Teachers Nutrition Habits and Physical Activity Levels in the Global (Covid-19) of Education and Training, 7(1): 51-59

History:

Received: 24 November 2020

Revised: 28 December 2020

Accepted: 10 January 2021

Published: 2 February 202

Licensed: This work is licensed under a Creative Commons

Attribution 3.0 License $(\mathrm{cc}) \mathbf{E Y}$

Publisher: Asian Online Journal Publishing Group
Acknowledgement: All authors contributed to the conception and design of the study.

Funding: This study received no specific financial support.

Competing Interests: The authors declare that they have no conflict of interests.

Transparency: The authors confirm that the manuscript is an honest, accurate, and transparent account of the study was reported; that no vital features of the study have been omitted; and that any discrepancies from the study as planned have been explained.

Ethical: This study follows all ethical practices during writing.

\section{Contents}

1. Introduction

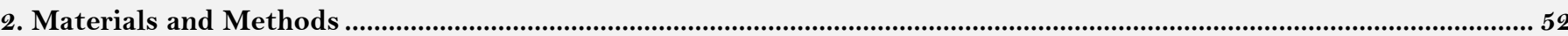

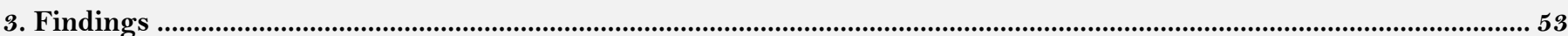

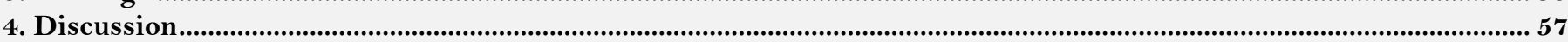

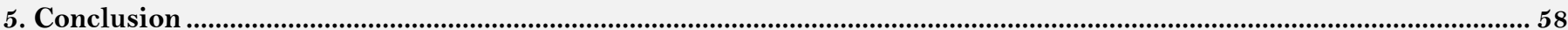

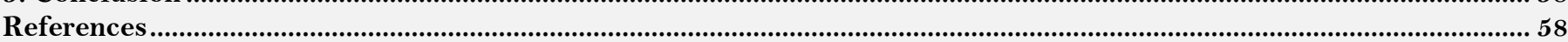




\section{Contribution of this paper to the literature}

This study contributes to existing literature by determining Physical Education and Sports teachers' nutritional habits and physical activity levels during the global epidemic (covid-19) process.

\section{Introduction}

The corona virus (Covid-19) appeared in Wuhan, China in December 2019, and despite all the measures taken by the Chinese government to stop this epidemiological phenomenon, it could not prevent the spread of the infection to the world. In this context, a number of difficulties have arisen in the world that have changed the usual life due to the Covid-19 epidemic (World Health Organization, 2020). Many countries have adapted to the new situation, especially social distance, mask, hygiene and home isolation have now become part of everyday life (Merriam-Webster, 2020; Miller, 2020).

With the measures taken to prevent and control the spread of the Covid-19 epidemic, a number of changes have occurred in eating habits, physical activity levels, consumer behavior, education and training methods and daily life. In order to prevent these negative changes in the life of societies, continuation of physical activity, sleep patterns, adequate and balanced nutrition are of great importance. Especially in the restrictions that exist with the quarantine period, regular physical activities as well as adequate and balanced nutrition are important in protecting the health of individuals. By encouraging all these factors (physical activity, adequate and balanced diet, social distance, etc.), it will be the most effective treatment method in protecting individuals and communities against the virus (Imboden et al., 2019; Lavie, Ozemek, Carbone, Katzmarzyk, \& Blair, 2019; Ozemek et al., 2018).

Nutrition is expressed as individuals' taking adequate amounts of nutrients and using them in the body in order to continue their lives in a more vigorous and productive way as well as growth and development. And adequate and balanced nutrition is defined as taking enough and needed amount of food to continue their lives in a healthy way and use it in the body. It is important to protect people from diseases together with the protection of physical fitness level of people with adequate and balanced nutrition (Bozkurt \& Erdoğan, 2019).

Physical activity is expressed as making a movement in the body as a result of people's energy expenditure (Cengiz \& Delen, 2019). Regular physical activities not only reduce stress and anxiety, but also affect the immune system positively (Nieman \& Wentz, 2019). Professional institutions and persons in the field stated that exercises for 150-300 minutes of moderate intensity activities or half of these times for high intensity per week, and balance and strength exercises involving large muscle groups 2 days a week would positively contribute to individuals' physical fitness levels as well as maintaining their health (Demirel \& Kayıhan, 2014; Piepoli et al., 2016; Piercy, Troiano, \& Ballard, 2018). They stated that people who regularly engaged in physical activity could reduce the risk of acute respiratory distress syndrome, which is an important cause of death in patients with corona virus (COVID19, 2020). In addition to the sedentary lifestyle that emerged as a result of the epidemic period, inadequate and unbalanced nutrition also increases the risk of cardiovascular diseases, obesity and Covid-19.

The Covid-19 epidemic affects the way of life of societies, as well as making it difficult by negatively affecting the lifestyles of physical education and sports teachers, who previously had a more active lifestyle. The responsibility of individuals to support the immune system during the epidemic period has been stated as adopting a healthy lifestyle, eating adequate and balanced nutrition, paying attention to sleep patterns and maintaining regular physical activities (Naja \& Hamadeh, 2020). In addition, individuals are recommended to avoid smoking and alcohol and to minimize stress (Harvard Medical School, 2020). It is stated that individuals who cannot isolate themselves and do not regularly implement a physical activity program are at risk during the epidemic period (Centers for Disease Control and Prevention, 2020).

In this context, for physical education and sports teachers to continue their lives in a healthy way during the global epidemic process depends on regular physical activity as well as adequate and balanced nutrition during the quarantine process. This research was conducted to determine the nutritional habits and physical activity levels of physical education and sports teachers in the covid-19 process.

\section{Materials and Methods}

Participants: The population of the study consisted of physical education and sports teachers working in Elazı $\breve{g}$ province, and the sample group consisted of 156 (121 male, 35 female) physical education and sports teachers who voluntarily participated in the study.

Data Collection Tools: Questionnaire method, one of the data collection techniques, was used in the study. In obtaining the data: A "Personal Information Form" consisting of 7 questions to determine the demographic information of the participants was applied. To determine the nutritional habits, a questionnaire consisting of 25 questions by Yücel (2015) aiming to examine the Nutrition Habits and Nutrition Knowledge Levels of Healthcare Professionals was applied. And the short form of the "International Physical Activity Questionnaire" was adapted according to the purpose of the study and applied to the participants in the process of obtaining the data.

\subsection{MET (Metabolic Equivalence) Calculation}

$1 \mathrm{MET}=3.5 \mathrm{ml} / \mathrm{kg} / \mathrm{min}$. oxygen consumption

METx3.5x weight $(\mathrm{kg}) / 200=\ldots \mathrm{kcal} / \mathrm{min}$.

According to the physical activity score, the physical activity levels of the participants are classified as "low, medium and high" (Devran \& Saka, 2019):

- $\quad<$ MET mild severe activity.

- 3-6 MET moderate severe activity.

- $\quad>6$ MET high intensity activity.

Statistical Analysis: In the analysis of the data, SPSS package program was used and the data determined to show normal distribution by looking at the normality test analysis of "Kolmogorov-Smirnov" values, were applied 
Independent Samples $\mathrm{t}$ and One-Way Anova test for in-group comparisons. Significance level was accepted as $\mathrm{p}$ $<0.05$.

\section{Findings}

Table-1. Demographic information of physical education teachers.

\begin{tabular}{|c|c|c|c|}
\hline & & Frequency & Percent (\%) \\
\hline \multirow[t]{2}{*}{ Gender } & Male & 121 & 77.6 \\
\hline & Female & 35 & 22.4 \\
\hline \multirow[t]{5}{*}{ Age } & $23-27$ & 19 & 12.2 \\
\hline & $28-32$ & 25 & 16 \\
\hline & $33-37$ & 37 & 23.7 \\
\hline & $38-42$ & 32 & 20.5 \\
\hline & 43 and over & 43 & 27.6 \\
\hline \multirow[t]{3}{*}{ Marital status } & Married & 46 & 29.5 \\
\hline & Single & 105 & 67.3 \\
\hline & Divorced & 5 & 3.2 \\
\hline \multirow[t]{4}{*}{ Size } & $150-160$ & 6 & 3.8 \\
\hline & $161-170$ & 35 & 22.4 \\
\hline & $171-180$ & 92 & 59 \\
\hline & $181-190$ & 23 & 14.7 \\
\hline \multirow[t]{5}{*}{ Weight } & $51-60$ & 13 & 8.3 \\
\hline & $61-70$ & 27 & 17.3 \\
\hline & $71-80$ & 60 & 38.5 \\
\hline & $81-90$ & 36 & 23.1 \\
\hline & 91 and over & 20 & 12.8 \\
\hline \multirow[t]{5}{*}{ Professional Seniority } & $1-5$ & 38 & 24.4 \\
\hline & $6-10$ & 43 & 27.6 \\
\hline & $11-15$ & 27 & 17.3 \\
\hline & $16-20$ & 22 & 14.1 \\
\hline & 21 and over & 26 & 16.7 \\
\hline \multirow[t]{5}{*}{ Income status } & $3000-4000$ & 32 & 20.5 \\
\hline & $4001-5000$ & 67 & 42.9 \\
\hline & $5001-6000$ & 37 & 23.7 \\
\hline & $6001-7000$ & 9 & 5.8 \\
\hline & 7001 and over & 11 & 7.1 \\
\hline \multirow[t]{2}{*}{ Nutrition Education } & Yes & 17 & 10.9 \\
\hline & No & 139 & 89.1 \\
\hline \multirow[t]{2}{*}{ Chronic Disease } & Yes & 97 & 62.2 \\
\hline & No & 59 & 37.8 \\
\hline
\end{tabular}

3.1. Physical Education and Sports Teachers' Pre-Pandemic Nutritional Habits and Physical Activity Levels Frequency Analysis

Table 2. Physical education teachers' nutritional knowledge levels.

\begin{tabular}{|c|c|c|c|}
\hline & & Frequency & Percent (\%) \\
\hline \multirow{4}{*}{ Number of main meals per day } & 1 meal & - & - \\
\hline & 2 meals & 83 & 53.2 \\
\hline & 3 meals & 73 & 46.8 \\
\hline & More & - & - \\
\hline \multirow{4}{*}{ Skipping meals during the day } & Breakfast & 15 & 9.6 \\
\hline & Lunch & 81 & 51.9 \\
\hline & Dinner & 6 & 3.8 \\
\hline & No skip & 54 & 34.6 \\
\hline \multirow{6}{*}{ Reasons for skipping meals } & Changes in eating habits & 22 & 14.1 \\
\hline & For not wanting to & 31 & 19.9 \\
\hline & Gaining weight & 12 & 7.7 \\
\hline & Stress & 17 & 10.9 \\
\hline & Changes in sleeping habit & 14 & 9 \\
\hline & Being busy & 60 & 38.5 \\
\hline \multirow{5}{*}{$\begin{array}{l}\text { The most common types of food / drink consumed between } \\
\text { meals }\end{array}$} & Carbonated / Acid drinks & 12 & 7.7 \\
\hline & Juice etc. & 23 & 14.7 \\
\hline & Cake / Cookie / Biscuit & 33 & 21.2 \\
\hline & Candy / Chocolate etc. & 16 & 10.3 \\
\hline & Fruit / Dried fruit etc & 72 & 46.2 \\
\hline \multirow{5}{*}{ Daily water consumption } & 1 liter and less & 30 & 19.2 \\
\hline & 1.5 liters & 52 & 33.3 \\
\hline & 2 liters & 29 & 18.6 \\
\hline & 2.5 liters & 19 & 12.2 \\
\hline & 3 liters and more & 26 & 16.7 \\
\hline
\end{tabular}

When Table 1 is examined, it is seen that the physical education and sports teachers; gender, age, marital status, height, weight, professional seniority and income status respectively determined as follows: 
$77.6 \%$ are men, $22.4 \%$ are women, 43 people are 43 years old and over, 37 people are $33-37$ years old, 32 people are $38-42$ years old, 25 people are $28-32$ years old and 19 people are $23-27$ years old $67.3 \%$ are married, $29.5 \%$ are single, $3.2 \%$ are divorced, 92 persons are between $171-180 \mathrm{~cm}, 35$ persons are between $161-170 \mathrm{~cm}, 23$ persons are between $181-190 \mathrm{~cm}, 6$ persons are between $150-160 \mathrm{~cm}, 60$ persons are between 71-80 kg, 36 persons are between $81-90 \mathrm{~kg}, 27$ persons are between 61-70 kg, 20 persons are $90 \mathrm{~kg}$ and above, 13 persons are between $51-60 \mathrm{~kg}, 43$ persons have seniority between 6-10 years, 38 persons 1-5 years, 27 persons 11-15 years, 26 persons 21 years and above, 22 persons 16-20 years, 67 persons have an income level of between 4001-5000 TL, 37 persons 5001-6000 TL, 32 persons 3000-4000 TL, 11 persons $7001 \mathrm{TL}$ and above, and 9 people had an income level of 6001-7000 TL. In addition, $89.1 \%$ of the participants were found not to receive nutrition training and $62.2 \%$ of them had a chronic disease.

According to Table 2 it has been determined that $46.8 \%$ of the physical education and sports teachers participating in the study consume three main meals a day, $51.9 \%$ do not regularly eat lunch, $38.5 \%$ skip meals due to workload, $46.2 \%$ mostly have fruit / dried fruit etc. in snacks, and 33.3\% consume 2 liters of water.

Table-3. Physical education teachers' physical activity levels

\begin{tabular}{|c|c|c|c|}
\hline & & Frequency & Percent (\%) \\
\hline & Yes & 60 & 38.5 \\
\hline \multirow[t]{3}{*}{ Do you do physical activity regularly? } & No & 30 & 19.2 \\
\hline & Not totally & 66 & 42.3 \\
\hline & None & 15 & 9.6 \\
\hline \multirow[t]{7}{*}{ How many days do you exercise in a week? } & 1 day & 16 & 10.3 \\
\hline & 2 days & 32 & 20.5 \\
\hline & 3 days & 55 & 35.3 \\
\hline & 3 days or more & 38 & 24.4 \\
\hline & At home & 47 & 30.1 \\
\hline & Jogging / Walking Areas & 53 & 34 \\
\hline & Sports Areas of the Municipality & 35 & 22.4 \\
\hline Where do you do your physical activities? & Sports Halls & 21 & 13.5 \\
\hline \multirow{2}{*}{$\begin{array}{l}\text { How many hours / minutes do you exercise per } \\
\text { week? }\end{array}$} & & & \\
\hline & Minute / average & \multicolumn{2}{|c|}{240.22} \\
\hline
\end{tabular}

When Table 3 was examined it was found that physical education and sports teachers who participated in the study; $42.3 \%$ of them partially participated in a physical activity program, $35.3 \%$ did physical activity three days a week and $30.1 \%$ did physical activity at home.

\subsection{Physical Education and Sports Teachers' Nutritional Habits and Physical Activity Levels Frequency Analysis during Pandemic Period}

Table-4. Physical education teachers' nutritional knowledge levels.

\begin{tabular}{|c|c|c|c|}
\hline & & Frequency & Percent (\%) \\
\hline \multirow{3}{*}{$\begin{array}{l}\text { Has there been any change in the nutrition habits during } \\
\text { the pandemic period? }\end{array}$} & Yes & 67 & 42.9 \\
\hline & No & 46 & 29.5 \\
\hline & Partly & 43 & 27.6 \\
\hline \multirow{3}{*}{ Number of main meals per day } & 1 meal & 69 & 44.2 \\
\hline & 2 meals & 70 & 44.9 \\
\hline & 3 meals & 17 & 10.9 \\
\hline \multirow[t]{4}{*}{ Skipping meals during the day } & Breakfast & 15 & 9.6 \\
\hline & Lunch & 80 & 51.3 \\
\hline & Dinner & 48 & 30.8 \\
\hline & No skip & 13 & 8.3 \\
\hline \multirow{3}{*}{$\begin{array}{l}\text { Has there been any change in the type of food / beverage } \\
\text { for the snack you consume during the pandemic? }\end{array}$} & Yes & 47 & 30.1 \\
\hline & No & 63 & 40.4 \\
\hline & Partially & 46 & 29.5 \\
\hline \multirow{6}{*}{ Reasons for skipping meals } & Changes in eating habits & 22 & 14.1 \\
\hline & For not wanting to & 19 & 12.2 \\
\hline & Gaining weight & 13 & 8.3 \\
\hline & Stress & 65 & 41.7 \\
\hline & Changes in sleeping habit & 28 & 17.9 \\
\hline & Being busy & 9 & 5.8 \\
\hline \multirow{2}{*}{$\begin{array}{l}\text { Has there been a change in the number of daily main meals } \\
\text { during the pandemic process? }\end{array}$} & Yes & 69 & 44.2 \\
\hline & No & 87 & 55.8 \\
\hline \multirow{3}{*}{ Has there been a change in daily fluid consumption? } & Increased & 53 & 34 \\
\hline & Decreased & 33 & 21.2 \\
\hline & Unchanged & 70 & 44.9 \\
\hline \multirow{6}{*}{$\begin{array}{l}\text { The most common types of food / drink consumed between } \\
\text { meals }\end{array}$} & Carbonated / Acid drinks & 15 & 9.6 \\
\hline & Juice etc. & 19 & 12.2 \\
\hline & Cake / Cookie / Biscuit & 38 & 24.4 \\
\hline & Candy / Chocolate etc. & 19 & 12.2 \\
\hline & Fruit / Dried fruit etc. & 52 & 33.3 \\
\hline & Carbonated / Acid drinks & 13 & 8.3 \\
\hline \multirow{5}{*}{ Daily water consumption } & 1 liter and less & 31 & 19.9 \\
\hline & 1.5 liters & 39 & 25 \\
\hline & 2 liters & 33 & 21.2 \\
\hline & 2.5 liters & 21 & 13.5 \\
\hline & 3 liters and more & 32 & 20.5 \\
\hline
\end{tabular}


When Table 4 is examined, it is observed that $42.9 \%$ of the physical education and sports teachers participating in the study have changes in their eating habits, $44.9 \%$ consume two main meals a day and the most skipped main meal is lunch with $51.3 \%$ and $41 \% 7$ of them skip meals due to stress.

Table-5. Physical education teachers' physical activity levels

\begin{tabular}{|c|c|c|c|}
\hline & & Frequency & Percent (\%) \\
\hline \multirow{3}{*}{ Do you do physical activity regularly? } & Yes & 58 & 37.2 \\
\hline & No & 49 & 31.4 \\
\hline & Partially & 49 & 31.4 \\
\hline \multirow{4}{*}{ How many days do you exercise a week? } & 1 day & 21 & 13.5 \\
\hline & 2 days & 54 & 34.6 \\
\hline & 3 days & 49 & 31.4 \\
\hline & 3 days or more & 32 & 20.5 \\
\hline \multirow{3}{*}{$\begin{array}{l}\text { Has there been a negative change in your } \\
\text { physical activity status? }\end{array}$} & Yes & 67 & 42.9 \\
\hline & No & 19 & 12.2 \\
\hline & Partially & 70 & 44.9 \\
\hline \multirow{4}{*}{ Where do you do your physical activities? } & At home & 103 & 66 \\
\hline & Jogging / Walking Areas & 23 & 14.7 \\
\hline & Sports Areas of the Municipality & 12 & 7.7 \\
\hline & Sports Halls & 18 & 11.5 \\
\hline \multirow{3}{*}{ Do you believe home exercises are helpful? } & Yes & 85 & 54.5 \\
\hline & No & 20 & 12.8 \\
\hline & Partially & 51 & 32.7 \\
\hline \multirow{3}{*}{$\begin{array}{l}\text { Has the State's Stay Home Project affected } \\
\text { your exercise? }\end{array}$} & Yes & 58 & 37.2 \\
\hline & No & 74 & 47.4 \\
\hline & Partially & 24 & 15.4 \\
\hline \multirow{3}{*}{ Has your body weight changed? } & Increased & 84 & 53.8 \\
\hline & Decreased & 32 & 20.5 \\
\hline & Unchanged & 40 & 25.6 \\
\hline $\begin{array}{l}\text { How many hours / minutes do you exercise } \\
\text { per week? }\end{array}$ & Minute / average & 132.24 & \\
\hline
\end{tabular}

Looking Table 5, it was determined that the majority of physical education and sports teachers participating in the study did not participate in a physical activity program regularly, 34.6\% did physical activity two days a week, $53.8 \%$ had an increase in their body weight and $66 \%$ did physical activity at home. In addition, $47.4 \%$ of respondents expressed no, 37.2\% yes and $15.4 \%$ partially on the item "Was "Stay at Home" project of the state effective in your physical activity?"

\subsection{Physical Education and Sports Teachers' Pre-Pandemic Nutrition Habits and Physical Activity Levels t and Variance Analysis}

Table-6. Physical education and sports teachers' analysis of nutritional habits and nutritional knowledge levels $\mathrm{t}$ test according to their demographic information.

\begin{tabular}{|c|c|c|c|c|c|c|c|c|c|}
\hline & & \multicolumn{2}{|c|}{ Nutrition } & \multirow[b]{2}{*}{$\mathbf{t}$} & \multirow[b]{2}{*}{$\mathbf{p}$} & \multicolumn{2}{|c|}{ Physical Activity } & \multirow[b]{2}{*}{$\mathbf{t}$} & \multirow[b]{2}{*}{$\mathbf{p}$} \\
\hline & & $\bar{X}$ & Sd & & & $\bar{X}$ & Sd. & & \\
\hline \multirow{2}{*}{ Gender } & Female & 13.57 & 5.04 & \multirow[b]{2}{*}{56.831} & \multirow[b]{2}{*}{0.00} & 7.01 & 2.26 & \multirow{2}{*}{-65.077} & \multirow[b]{2}{*}{0.00} \\
\hline & Male & 22.28 & 1.27 & & & 12.00 & 0.00 & & \\
\hline \multirow{2}{*}{$\begin{array}{l}\text { Nutrition } \\
\text { Education }\end{array}$} & Yes & 6.64 & 0.70 & \multirow[t]{2}{*}{77.473} & \multirow[t]{2}{*}{0.00} & 5.29 & 2.05 & \multirow{2}{*}{-3.822} & \multirow{2}{*}{0.05} \\
\hline & No & 16.61 & 5.15 & & & 8.48 & 2.78 & & \\
\hline \multirow{2}{*}{$\begin{array}{l}\text { Chronic } \\
\text { Disease }\end{array}$} & Yes & 11.68 & 3.67 & \multirow[t]{2}{*}{44.055} & \multirow[t]{2}{*}{0.00} & 6.49 & 2.23 & \multirow{2}{*}{15.435} & \multirow{2}{*}{0.12} \\
\hline & No & 21.84 & 1.21 & & & 10.83 & 1.44 & & \\
\hline
\end{tabular}

Note: $\mathrm{p}<.05$

Looking at the Tables 6 and 7, It was determined that there was a statistically significant difference between the mean scores of physical education and sports teachers participating in the study from their nutritional habits and physical activity levels according to gender, marital status, educational status, profession, age, height, weight, nutritional education and income, while no statistically significant difference was found according to the chronic disease status.

\subsection{Physical Education and Sports Teachers' Nutritional Habits and Physical Activity Levels $t$ and Variance Analysis during Pandemic Period}

When Tables 8 and 9 are evaluated, it has been determined that there is a statistical difference between the mean scores of dietary habits and physical activity levels of physical education and sports teachers participating in the study according to their gender, marital status, educational status, profession, age, height, weight, nutritional education, chronic disease status and income.

\subsection{Physical Activity Levels $t$ Test Analyzes of Physical Education and Sports Teachers in and before the Pandemic Period}

When Table 10 is examined, it has been determined that there is a statistically significant difference between the physical activity levels of physical education and sports teachers participating in the study during and before the pandemic period. 


\begin{tabular}{|c|c|c|c|c|c|c|c|c|c|}
\hline & & \multicolumn{2}{|c|}{ Nutrition } & \multirow[b]{2}{*}{$\mathbf{F}$} & \multirow[b]{2}{*}{ Sig } & \multicolumn{2}{|c|}{ Physical Activity } & \multirow[b]{2}{*}{$\mathbf{F}$} & \multirow[b]{2}{*}{ Sig } \\
\hline & & $\bar{X}$ & Sd & & & $\bar{X}$ & Sd. & & \\
\hline \multirow{5}{*}{ Age } & $23-27$ & 6.78 & 0.78 & \multirow{5}{*}{740.47} & \multirow[b]{5}{*}{0.00} & 5.26 & 1.93 & \multirow{5}{*}{228.74} & \multirow{5}{*}{0.00} \\
\hline & $28-32$ & 9.96 & 1.17 & & & 5.00 & 0.00 & & \\
\hline & $33-37$ & 12.67 & 1.31 & & & 6.32 & 0.74 & & \\
\hline & $38-42$ & 19.34 & 1.69 & & & 9.84 & 1.13 & & \\
\hline & 43 or over & 22.23 & 1.15 & & & 11.51 & 1.05 & & \\
\hline \multirow{4}{*}{ Height } & $150-160 \mathrm{~cm}$ & 6.00 & 0.00 & \multirow[b]{4}{*}{94.58} & \multirow[b]{4}{*}{0.00} & 6.33 & 1.63 & \multirow{4}{*}{67.956} & \multirow{4}{*}{0.00} \\
\hline & $161-170 \mathrm{~cm}$ & 8.82 & 1.65 & & & 4.91 & 1.24 & & \\
\hline & $171-180 \mathrm{~cm}$ & 17.00 & 4.27 & & & 8.51 & 2.30 & & \\
\hline & $181-190 \mathrm{~cm}$ & 22.30 & 1.55 & & & 12.00 & 0.00 & & \\
\hline \multirow{5}{*}{ Weight } & $51-60 \mathrm{~kg}$ & 6.38 & 0.50 & \multirow{5}{*}{265.46} & \multirow{5}{*}{0.00} & 4.84 & 2.07 & \multirow{5}{*}{75.040} & \multirow{5}{*}{0.00} \\
\hline & $61-70 \mathrm{~kg}$ & 9.29 & 1.38 & & & 5.25 & 0.94 & & \\
\hline & $71-80 \mathrm{~kg}$ & 14.31 & 2.96 & & & 7.53 & 2.18 & & \\
\hline & $81-90 \mathrm{~kg}$ & 21.80 & 0.78 & & & 10.33 & 1.45 & & \\
\hline & $91 \mathrm{~kg}$ or over & 22.20 & 1.64 & & & 12.00 & 0.00 & & \\
\hline \multirow{3}{*}{$\begin{array}{l}\text { Marital } \\
\text { Status }\end{array}$} & Married & 18.31 & 4.32 & \multirow{3}{*}{110.02} & \multirow{3}{*}{0.00} & 5.13 & 1.30 & \multirow{3}{*}{71.751} & \multirow{3}{*}{0.00} \\
\hline & Single & 8.69 & 1.90 & & & 9.26 & 2.37 & & \\
\hline & Divorced & 19.80 & 1.78 & & & 12.00 & 0.00 & & \\
\hline \multirow{5}{*}{$\begin{array}{l}\text { Professional } \\
\text { Seniority }\end{array}$} & $1-5$ years & 8.21 & 1.74 & \multirow{5}{*}{595.465} & \multirow{5}{*}{0.00} & 5.13 & 1.43 & \multirow{5}{*}{226.091} & \\
\hline & $6-10$ years & 12.44 & 1.35 & & & 6.13 & 0.83 & & \\
\hline & 11-15 years & 18.96 & 1.55 & & & 10.00 & 1.17 & & \\
\hline & 16-20 years & 21.86 & 0.35 & & & 10.36 & 1.43 & & 0.00 \\
\hline & $\begin{array}{l}21 \text { years or } \\
\text { over }\end{array}$ & 22.38 & 1.47 & & & 12.00 & 0.00 & & \\
\hline & $3001-4000$ & 7.68 & 1.35 & & & 5.06 & 1.54 & & \\
\hline & $4001-5000$ & 13.83 & 2.91 & & & 7.25 & 2.18 & & \\
\hline Income Status & $5001-6000$ & 21.75 & 0.83 & & & 10.29 & 1.45 & 67.150 & 0.00 \\
\hline & $6001-7000$ & 23.00 & 0.00 & & & 12.00 & 0.00 & & \\
\hline & 7001 or over & 21.54 & 2.01 & 248.966 & 0.00 & 12.00 & 0.00 & & \\
\hline
\end{tabular}

Note: $\mathrm{p}<.05$

Table-8. Physical education and sports teachers' nutritional habits and physical activity levels $t$ test analyzes according to the demographic information.

\begin{tabular}{|c|c|c|c|c|c|c|c|c|c|}
\hline & & \multicolumn{2}{|c|}{ Nutrition } & \multirow[b]{2}{*}{$\mathbf{t}$} & \multirow[b]{2}{*}{ p } & \multicolumn{2}{|c|}{ Physical Activity } & \multirow[b]{2}{*}{$\mathbf{t}$} & \multirow[b]{2}{*}{$\mathbf{p}$} \\
\hline & & $\bar{X}$ & Sd & & & $\bar{X}$ & Sd. & & \\
\hline \multirow{2}{*}{ Gender } & Female & 18.45 & 6.84 & \multirow[b]{2}{*}{-11.131} & \multirow[b]{2}{*}{0.00} & 6.22 & 2.40 & \multirow[b]{2}{*}{-6.281} & \multirow[b]{2}{*}{0.00} \\
\hline & Male & 31.54 & 2.16 & & & 8.85 & 1.08 & & \\
\hline \multirow{2}{*}{$\begin{array}{l}\text { Nutrition } \\
\text { Education }\end{array}$} & Yes & 9.23 & 0.66 & \multirow[b]{2}{*}{-7.552} & \multirow[b]{2}{*}{0.00} & 4.00 & 0.00 & \multirow{2}{*}{-5.488} & \multirow{2}{*}{0.00} \\
\hline & $\mathrm{No}$ & 22.87 & 7.42 & & & 7.15 & 2.36 & & \\
\hline \multirow{2}{*}{$\begin{array}{l}\text { Chronic } \\
\text { Disease }\end{array}$} & Yes & 16.14 & 5.58 & \multirow[b]{2}{*}{-17.959} & \multirow[t]{2}{*}{0.00} & 6.05 & 2.48 & \multirow{2}{*}{-5.446} & \multirow{2}{*}{0.00} \\
\hline & No & 30.01 & 2.55 & & & 8.06 & 1.76 & & \\
\hline
\end{tabular}

Note: $\mathrm{p}<.05$

Table-9. Physical education teachers' nutrition habits and physical activity levels variance analysis according to demographic information.

\begin{tabular}{|c|c|c|c|c|c|c|c|c|c|}
\hline & & \multicolumn{2}{|c|}{ Nutrition } & \multirow[b]{2}{*}{$\mathbf{F}$} & \multirow{2}{*}{ Sig } & \multicolumn{2}{|c|}{ Physical Activity } & \multirow[b]{2}{*}{$\mathbf{F}$} & \multirow[b]{2}{*}{ Sig } \\
\hline & & $\bar{X}$ & Sd & & & $\bar{X}$ & Sd & & \\
\hline \multirow{5}{*}{ Age } & $23-27$ & 9.42 & 0.83 & \multirow[b]{5}{*}{521.459} & \multirow[b]{5}{*}{0.00} & 4.00 & 0.00 & \multirow{5}{*}{53.741} & \multirow{5}{*}{0.00} \\
\hline & $28-32$ & 12.40 & 0.86 & & & 4.36 & 0.48 & & \\
\hline & $33-37$ & 18.48 & 3.25 & & & 8.18 & 2.54 & & \\
\hline & $38-42$ & 25.87 & 1.71 & & & 6.21 & 1.73 & & \\
\hline & 43 or over & 31.06 & 2.18 & & & 8.74 & 1.07 & & \\
\hline \multirow{4}{*}{ Height } & $150-160 \mathrm{~cm}$ & 9.00 & 0.00 & \multirow[b]{4}{*}{155.360} & \multirow[b]{4}{*}{0.00} & 4.00 & 0.00 & \multirow{4}{*}{45.458} & \multirow{4}{*}{0.00} \\
\hline & $161-170 \mathrm{~cm}$ & 11.22 & 1.47 & & & 4.17 & 0.38 & & \\
\hline & $171-180 \mathrm{~cm}$ & 23.19 & 5.18 & & & 7.46 & 2.23 & & \\
\hline & $181-190 \mathrm{~cm}$ & 32.86 & 1.35 & & & 8.95 & 1.10 & & \\
\hline \multirow{5}{*}{ Weight } & $51-60 \mathrm{~kg}$ & 9.00 & 0.00 & \multirow[b]{5}{*}{280.095} & \multirow[b]{5}{*}{0.00} & 4.00 & 0.00 & \multirow{5}{*}{32.233} & \multirow{5}{*}{0.00} \\
\hline & $61-70 \mathrm{~kg}$ & 11.74 & 1.05 & & & 4.18 & 0.39 & & \\
\hline & $71-80 \mathrm{~kg}$ & 20.21 & 4.38 & & & 7.28 & 2.45 & & \\
\hline & $81-90 \mathrm{~kg}$ & 28.52 & 1.15 & & & 7.91 & 1.82 & & \\
\hline & $91 \mathrm{~kg}$ or over & 33.15 & 1.22 & & & 8.80 & 1.10 & & \\
\hline \multirow{3}{*}{ Marital Status } & Married & 25.23 & 5.54 & \multirow[b]{3}{*}{162.316} & \multirow[b]{3}{*}{0.00} & 7.84 & 2.14 & \multirow{3}{*}{68.664} & \multirow{3}{*}{0.00} \\
\hline & Single & 11.23 & 1.77 & & & 4.23 & 0.43 & & \\
\hline & Divorced & 34.00 & 0.00 & & & 34.00 & 0.00 & & \\
\hline \multirow{5}{*}{$\begin{array}{l}\text { Professional } \\
\text { Seniority }\end{array}$} & $1-5$ years & 10.73 & 1.51 & \multirow[b]{5}{*}{481.558} & \multirow[b]{5}{*}{0.00} & 4.10 & 0.31 & \multirow{5}{*}{51.118} & \\
\hline & $6-10$ years & 17.79 & 3.48 & & & 7.72 & 2.63 & & \\
\hline & 11-15 years & 25.55 & 1.67 & & & 5.70 & 1.29 & & \\
\hline & $16-20$ years & 28.68 & 0.64 & & & 8.77 & 0.92 & & 0.00 \\
\hline & 21 years or over & 32.42 & 1.79 & & & 8.76 & 1.17 & & \\
\hline & $3001-4000$ & 10.37 & 1.36 & & & 4.12 & 0.33 & & \\
\hline & $4001-5000$ & 19.22 & 4.71 & & & 6.94 & 2.52 & & \\
\hline Income Status & $5001-6000$ & 28.48 & 1.16 & & & 7.83 & 1.86 & 23.776 & 0.00 \\
\hline & $6001-7000$ & 32.11 & 1.16 & & & 9.44 & 0.88 & 23.160 & 0.00 \\
\hline & 7001 or over & 34.00 & 0.00 & 210.333 & 0.00 & 8.27 & 1.00 & & \\
\hline
\end{tabular}




\begin{tabular}{|c|c|c|c|c|c|}
\hline & & $\bar{X}$ & Sd & $\mathbf{t}$ & p \\
\hline \multirow{2}{*}{ Physical Activity } & Before Pandemic & 7.53 & 2.49 & \multirow[b]{2}{*}{24.604} & \multirow[t]{2}{*}{0.00} \\
\hline & Pandemic Period & 6.81 & 2.44 & & \\
\hline \multirow{2}{*}{ Nutrition } & Before Pandemic & 15.52 & 5.77 & \multirow[b]{2}{*}{-3.503} & \multirow[b]{2}{*}{0.01} \\
\hline & Pandemic Period & 21.39 & 8.20 & & \\
\hline
\end{tabular}

Note: $\mathrm{p}<.05$

\section{Discussion}

When the eating habits of physical education and sports teachers before and during the pandemic are examined in the study; Before the pandemic, $53.2 \%$ of the teachers stated that the number of main meals per day was two, $51.9 \%$ stated that the most skipped main meal during the day was lunch, $38.5 \%$ of them said that the reason for skipping meals was work intensity, $46.2 \%$ of them stated that they consume fruit / dried fruit etc. between meals and $33.3 \%$ of them stated that they consume 1.5 liters of water per day. During the pandemic period, $44.2 \%$ of the teachers stated that the number of their main meals per day was two, 51.3\% stated that the most skipped main meal during the day was lunch, $41.7 \%$ stated that the reason for skipping meals was stress, $33 \%$ of them stated that they consume fruit/dried fruit etc between meals and $25 \%$ of them stated that they consume 1.5 liters of water a day. It was observed that most of the physical education and sports teachers participating in the study had a chronic illness and had not received any nutrition training before. In addition, it was also determined that the participants ' eating habits during and before the pandemic were not adequate and regular. In line with these results, it is seen that there are changes in the nutritional habits of physical education and sports teachers with the pandemic. Tïryaki, Pehlïvan, and Baba (2020) found that $14.7 \%$ of the participants were exercising at home and $54 \%$ ate two meals a day in the study, in which they examined the determination of the basic psychological needs of sports sciences students staying at home during the pandemic period. Mattioli, Sciomer, Cocchi, Maffei, and Gallina (2020) stated in their study that by encouraging activities such as healthy eating and regular physical activity during the pandemic period, people's risk of getting sick would decrease and the epidemic period would be overcome with minimum damage. Akyol and Celik (2020) found in their study that the nutritional habits of university students were negatively affected during the Covid-19 process. Khoramipour et al. (2020) in their study on physical activity and nutritional guidelines to help combat Covid-19, stated that a regular physical activity program and adequate and balanced nutrition will strengthen the immune system and have positive effects during the epidemic period. Mor, Acar, and Arslanoğlu (2020) stated in their compilation study that adequate and balanced nutrition would minimize the risk of developing diseases by strengthening the immune system. Garipoğlu and Bozar (2020) investigated the eating habits of individuals in social isolation in the Covid-19 epidemic and found that the participants' eating habits were irregular and their physical activity levels were low. Tek and Koçak (2020) stated in their research on the role of nutrition in supporting the immune system in the fight against coronavirus, that adequate and balanced nutrition will positively affect the immune system and help both the quality of life and the prevention of the epidemic. Dilber and Dilber (2020) found that the participants' eating habits were negatively affected during the epidemic period in the study in which they examined the effect of corona virus outbreak on individuals' eating habits. Ammar et al. (2020) in their study examining the effects of home isolation on nutrition and physical activity during the covid-19 period, stated that the pandemic period had a negative effect on physical activity and eating habits.

When the physical activity levels before and during the pandemic of the physical education and sports teachers participating in the study were evaluated, before the pandemic, $42.3 \%$ of teachers stated that they partly did a physical activity, $35.3 \%$ exercised three days a week and $34 \%$ did their physical activities in the running/walking areas. During the pandemic, $37.2 \%$ of teachers stated that they regularly did physical activity, $34.6 \%$ exercised two days a week and $66 \%$ said they did their physical activity at home. In addition, it was observed that the physical activity levels of the participants were insufficient during and before the pandemic. Tural (2020) in his study investigating the effect of physical activity on quality of life during pandemic period home quarantine, determined that the physical activity levels of the participants were low. Ercan and Keklicek (2020) stated that the physical activity levels of the participants were low in the research in which they examined the physical activity levels of university students due to the Covid-19 pandemic. Ozemek et al. (2018) stated in their study on Covid-19 and physical inactivity that physical activity would help prevent both Covid-19 and cardiovascular disorders that might occur in the organism. Cheval et al. (2020) stated in their study that doing enough physical activity and reducing the sedentary life span would help in coping with the Covid-19 epidemic. Pündük (2020) stated in his review study that regular exercises affect the immune system positively as well as protection against the virus and exercise can be recommended as a prescription. Woods et al. (2020) stated in their study that lower intensity and progressive exercises would strengthen the body's immune system and overcome the epidemic period with minimal damage, as high-intensity resistance exercises to be applied during the epidemic period would weaken the immune system. Arslan and Ercan (2020) studied the importance of exercise during the Covid-19 pandemic and social isolation process, stated that to overcome the pandemic process with minimal damage not only being active at home but also organizing exercise programs considering the physical characteristics of individuals would positively affect the health of individuals.

It was determined that there is a statistically significant difference in nutrition and physical activity levels of physical education and sports teachers participating in the study during and before the pandemic period $(p<0.05)$. While the average score of physical activity levels of physical education sports teachers before the pandemic is higher than the pandemic period, the average score of nutritional habits before the pandemic is lower than the pandemic period. It was observed that the participants paid more attention to their eating habits during the pandemic period. In addition, it was determined that the participants did not pay attention to their physical activity levels during the pandemic period. This may be due to home isolation and restrictions imposed to prevent the spread of the virus as a result of the pandemic. Reyes-Olavarría et al. (2020) in the study examining the nutrition 
and physical activity levels of individuals during the Covid-19 epidemic, stated that the Chilean people had changes in their eating habits, increase body weight and decrease in physical activity levels compared to the pre-epidemic period. Simşek, Koç, Ozsoy, and Karakuş (2020) found in their study that there was a statistical difference between physical activity levels before and after the pandemic. Sánchez-Sánchez et al. (2020) In their study, they examined the eating habits and physical activity levels of the Spaniards during and before the epidemic period. They stated that there were changes in their eating habits and they adopted an unhealthy eating habits such as alcoholic beverages, snacks and desserts, and their physical activity levels decreased compared to the pre-epidemic period.

\section{Conclusion}

As a result; during the pandemic period, it was determined that physical education and sports teachers' nutritional habits and physical activity levels were negatively affected. We believe that it will be beneficial to do physical activity regularly, as well as adequate and balanced nutrition, in order to overcome this new epidemic, which the whole world is struggling with today, with minimal damage. During the Covid-19 outbreak, activities such as information, programs, etc. can be arranged that will protect people's health, strengthen their immune systems, regulate their eating habits and increase their level of physical activity.

\section{References}

Akyol, P., \& Celik, A. (2020). Investigation of paramedic students' Nutritional Habits During the Covid-19 outbreak. Turkish Studies, 15(4), $25-37$.

Ammar, A., Brach, M., Trabelsi, K., Chtourou, H., Boukhris, O., Masmoudi, L., \& Müller, P. (2020). Tural E. Covid-19 the effect of physical activity level on quality of life in home quarantine during pandemic. Van Health Sciences Journal, 13, 10-18.

Arslan, E., \& Ercan, S. (2020). COVID-19 pandemic and the importance of exercise in social isolation process. Journal of Sports Medicine, 55(2), 188-191.

Bozkurt, E., \& Erdoğan, R. (2019). Investigation of eating habits of classroom teachers. OPUS International Journal of Society Studies, 13(19), 75-94.

Cengiz, S., \& Delen, B. (2019). Physical activity level in youth people International Journal of Current Educational Research, 5(2), $110-122$.

Centers for Disease Control and Prevention. (2020). Overweight \& obesity. Updated February 27, 2020. Retrieved from: https://www.cdc.gov/obesity/data/adult.html. USA. [Accessed September 24, 2020].

Cheval, B., Sivaramakrishnan, H., Maltagliati, S., Fessler, L., Forestier, C., Sarrazin, P., . . N Ntoumanis, N. (2020). Relationships between changes in self-reported physical activity, sedentary behaviour and health during the coronavirus (COVID-19) pandemic in France and Switzerland. Journal of Sports Sciences, 1-6.

COVID-19. (2020). COVID-19: Exercise may help prevent deadly [internet]. 2020 [updated 2020 April 14; cited 2020]. Retrieved from: https://newsroom.uvahealth.com/2020/04/15/covid-19-exercise-may-help-prevent-deadlycomplication/.

Demirel, H., \& Kayıhan, H. (2014). Turkey physical activity guide (2nd ed.). Ankara: Kuban Printing Publishing.

Devran, B. S., \& Saka, M. (2019). The effect of nutritional education given to high school students on nutritional habits, nutritional knowledge and physical activity. Nutrition and Diet Journal, 47(3), 5-14.

Dilber, A., \& Dilber, F. (2020). The coronavirus (COVID-19) outbreak on the eating habits of individuals. Journal of Tourism and Gastronomy Studies, 8(3), $2144-2162$.

Ercan, S., \& Keklïcek, H. (2020). Investigation of the change in physical activity levels of university students due to COVID-19 pandemic. Izmir Kâtip Celebi University Faculty of Health Sciences Journal, 5(2), 69-74.

Garipoğlu, G., \& Bozar, N. (2020). Changes in the eating habits of individuals in social isolation in the Covid-19 Outbreak. Pearson Journal Of Social Scrences \& Humantes, 6(6), 100-113.

Harvard Medical School. (2020). How to boost your immune system: Harvard Health Publishing. Retrieved from: https://www.health.harvard.edu/staying-healthy/how-to-boost-your-immunesystem.

Imboden, M. T., Harber, M. P., Whaley, M. H., Finch, W. H., Bishop, D. L., Fleenor, B. S., \& Kaminsky, L. A. (2019). The association between the change in directly measured cardiorespiratory fitness across time and mortality risk. Progress in Cardiovascular Diseases, $62(2), 157-162$.

Khoramipour, K., Basereh, A., Hekmatikar, A. A., Castell, L., Ruhee, R. T., \& Suzuki, K. (2020). Physical activity and nutrition guidelines to help with the fight against COVID-19. Journal of Sports Sciences, 39(1), 1-7.

Lavie, C. J., Ozemek, C., Carbone, S., Katzmarzyk, P. T., \& Blair, S. N. (2019). Sedentary behavior, exercise, and cardiovascular health. Circulation Research, 124(5), 799-815.

Mattioli, A., Sciomer, S., Cocchi, C., Maffei, S., \& Gallina, S. (2020). Quarantine during COVID-19 outbreak: Changes in diet and physical activity increase the risk of cardiovascular disease. Nutrition, Metabolism and Cardiovascular Diseases, 30(9), 1409-1411.

Merriam-Webster. (2020). Social distancing [internet] 2020. [updated 2020 July 15; cited 2020]. Avaible from: https://www.merriamwebster.com/dictionary/social\%20distancing.

Miller, K. (2020). Here's what a shelter in place order means during the Coronavirus Pandemic [internet]. 2020 [updated 2020 July 28 ; cited 2020]. Retrieved from: https://www.prevention.com/health/amp31738348/shelter-in-place-ordercoronavirus/.

Mor, A., Acar, K., \& Arslanoğlu, E. (2020). Covid-19 and nutrition approach in athletes. Journal of Physical Education and Sport Sciences, 22(2), $1-3$.

Naja, F., \& Hamadeh, R. (2020). Nutrition amid the COVID-19 pandemic: A multi-level framework for action. European Journal of Clinical Nutrition, 74(8), 1117-1121.Available at: https://doi.org/10.1038/s41430-020-0634-3.

Nieman, D., \& Wentz, L. (2019). The compelling link between physical activity and the body's defense system. Journal of Sport and Health Science, 8(3), 201-217.Available at: https://doi.org/10.1016/j.jshs.2018.09.009.

Ozemek, C., Laddu, D. R., Lavie, C. J., Claeys, H., Kaminsky, L. A., Ross, R., . . Blair, S. N. (2018). An update on the role of cardiorespiratory fitness, structured exercise and lifestyle physical activity in preventing cardiovascular disease and health risk. Progress in Cardiovascular Diseases, 61(5-6), 484-490.

Piepoli, M. F., Hoes, A., Agewall, S., Albus, C., Brotons, C., Catapano, A., . . Richter, D. (2016). European guidelines on cardiovascular disease prevention in clinical practice: The Sixth Joint Task Force of the European Society of Cardiology and Other Societies on Cardiovascular Disease Prevention in Clinical Practice (constituted by representatives of 10 societies and by invited experts)Developed with the special contribution of the European Association for Cardiovascular Prevention \& Rehabilitation (EACPR). European Heart Journal, 37(29), 2315-2381.Available at: https://doi.org/10.1093/eurheartj/ehw 106.

Piercy, K., Troiano, R., \& Ballard, R. (2018). The physical activity guidelines for Americans. JAMA, 320(19), 2020-2028.Available at: $10.1001 /$ jama.2018.14854.

Pündük, Z. (2020). Could the COVID-19 outbreak affect global trends, physical inactivity and sedentary behavior? Turkey Clinic of Sports Sciences, 12(2), 241-246.

Reyes-Olavarría, D., Latorre-Román, P. Á., Guzmán-Guzmán, I. P., Jerez-Mayorga, D., Caamaño-Navarrete, F., \& Delgado-Floody, P. (2020). Positive and negative changes in food habits, physical activity patterns, and weight status during COVID-19 confinement: associated factors in the Chilean population. International Journal of Environmental Research and Public Health, $17(15), 5431$.

Sánchez-Sánchez, E., Ramírez-Vargas, G., Avellaneda-López, Y., Orellana-Pecino, J. I., García-Marín, E., \& Díaz-Jimenez, J. (2020). Eating habits and physical activity of the Spanish population during the COVID-19 pandemic period. Nutrients, 12(9), 1-12.Available at: https://doi.org/10.3390/nu12092826. 
Simşek, E., Koç, K., Ozsoy, D., \& Karakuş, M. (2020). The title of the article: Investigation the effect of performing physical activity at home on sleep quality and life satisfaction during coronavirus (Covid-19) outbreak. International Journal of Applied Exercise Physiology, $9(7), 55-62$.

Tek, N. A., \& Koçak, T. (2020). The role of nutrition in supporting the immune system in combating coronavirus (Covid-19). Gazi Journal of Health Sciences, Special Issue, 18-45.

Tïryaki, K., Pehlïvan, M., \& Baba, K. H. (2020). Determining the basic psychological needs of sports sciences students staying at home within the scope of coronavirus (Covid-19) measures. Sports Education Journal, 4(3), 43-53.

Tural, E. (2020). Covid-19 the effect of physical activity level on quality of life in home quarantine during pandemic. Van Health Sciences Journal, 13(Special Issue)), 10-18.

Woods, J., Hutchinson, N., Powers, S., Roberts, W., Gomez-Cabrera, M., Radak, Z., \& Coelho-Júnior, H. (2020). The COVID-19 pandemic and physical activity. Sports Medicine and Health Science, 2(2), 55-64.

World Health Organization. (2020). Coronavirus disease (COVID-19) Pandemic [internet]. 2020 [updated 2020 May 5; cited 2019]. Retrieved from: https://www.who.int/emergencies/diseases/novel-coronavirus-2019.

Yücel, B. (2015). Examining the nutritional habits and nutritional knowledge levels of healthcare professionals. Master's Thesis, Başkent University Institute of Health Sciences. 